\title{
5-Hydroxymethylcytosine signatures in cell-free DNA provide information about tumor types and stages
}

\author{
Chun-Xiao Song ${ }^{1,13, *}$, Senlin Yin ${ }^{2,3, *}$, Li Ma ${ }^{4,5}$, Amanda Wheeler, ${ }^{5}$ Yu Chen ${ }^{2}$, Yan Zhang ${ }^{6}$, Bin Liu ${ }^{6,7}$, \\ Junjie Xiong ${ }^{8}$, Weihan Zhang ${ }^{9}$, Jiankun $\mathrm{Hu}^{9}$, Zongguang Zhou', Biao Dong ${ }^{2}$, Zhiqi Tian ${ }^{10}$, Stefanie S Jeffrey, \\ Mei-Sze Chua, ${ }^{4,5}$, Samuel $\mathrm{So}^{4,5}$, Weimin $\mathrm{Li}^{11}$, Yuquan Wei ${ }^{2}$, Jiajie Diao ${ }^{10}$, Dan Xie ${ }^{2,9,11}$, Stephen R Quake ${ }^{1,12}$ \\ ${ }^{1}$ Departments of Bioengineering and Applied Physics, Stanford University, Stanford, CA 94305, USA; ${ }^{2}$ State Key Laboratory of \\ Biotherapy, West China Hospital, Sichuan University and National Collaborative Innovation Center, Chengdu, Sichuan 610041, \\ China; ${ }^{3}$ Department of Neurosurgery, West China Hospital, Sichuan University, Chengdu, Sichuan 610041, China; ${ }^{4}$ Asian Liver \\ Center, Stanford University School of Medicine, Stanford, CA 94305, USA; ${ }^{5}$ Department of Surgery, Stanford University School \\ of Medicine, Stanford, CA 94305, USA; ${ }^{6}$ Department of Thoracic Oncology, West China Hospital, Sichuan University, Chengdu, \\ Sichuan 610041, China; ${ }^{7}$ Department of Pulmonary Tumor Ward, Sichuan Cancer Hospital, Chengdu, Sichuan 610041, China; \\ ${ }^{8}$ Department of Pancreatic Surgery, West China Hospital, Sichuan University, Chengdu, Sichuan 610041, China; ${ }^{9}$ Department of \\ Gastrointestinal Surgery, West China Hospital, Sichuan University, Chengdu, Sichuan 610041, China; ${ }^{10}$ Department of Cancer \\ Biology, University of Cincinnati College of Medicine, Cincinnati, OH 45267, USA; ${ }^{11}$ Center of Precision Medicine, West China \\ Hospital, Sichuan University, Chengdu, Sichuan 610041, China, ${ }^{12}$ Chan Zuckerberg Biohub, San Francisco, CA 94518, USA
}

5-Hydroxymethylcytosine $(5 \mathrm{hmC})$ is an important mammalian DNA epigenetic modification that has been linked to gene regulation and cancer pathogenesis. Here we explored the diagnostic potential of $5 \mathrm{hmC}$ in circulating cell-free DNA (cfDNA) using a sensitive chemical labeling-based low-input shotgun sequencing approach. We sequenced cellfree $5 \mathrm{hmC}$ from 49 patients of seven different cancer types and found distinct features that could be used to predict cancer types and stages with high accuracy. Specifically, we discovered that lung cancer leads to a progressive global loss of $5 \mathrm{hmC}$ in cfDNA, whereas hepatocellular carcinoma and pancreatic cancer lead to disease-specific changes in the cell-free hydroxymethylome. Our proof-of-principle results suggest that cell-free $5 \mathrm{hmC}$ signatures may potentially be used not only to identify cancer types but also to track tumor stage in some cancers.

Keywords: epigenetics; 5-hydroxylmethylcytosine; cell-free DNA; cancer diagnostics

Cell Research (2017) 27:1231-1242. doi:10.1038/cr.2017.106; published online 18 August 2017

\section{Introduction}

Circulating cell-free DNA (cfDNA) are DNA fragments found in the blood that originate from cell death in different tissues; this phenomenon has formed the basis of noninvasive prenatal diagnostic tests [1], organ trans-

\footnotetext{
*These two authors contributed equally to this work.

Correspondence: Stephen R Quake, Dan Xie

${ }^{a}$ Tel: +1 6507367890

E-mail: quake@stanford.edu

${ }^{\text {bE} E-m a i l: d a n x i e @ s c u . e d u . c n ~}$

${ }^{13}$ Current address: Ludwig Institute for Cancer Research and Target Discovery Institute, Nuffield Department of Medicine, University of Oxford, Oxford OX3 7FZ, UK

Received 17 July 2017; revised 23 July 2017; accepted 25 July 2017; published online 18 August 2017
}

plant rejection diagnostics [2], and cancer detection [3]. Recent work has focused on the identification of 5-methylcytosine $(5 \mathrm{mC})$ modifications in cfDNA to characterize a variety of potential health conditions [3-8]. However, there has been no investigation to date of alternative epigenetic DNA modifications in cfDNA, due in part to the challenges of identifying and sequencing alternative modifications in low-input DNA samples.

5 -Hydroxymethylcytosine $(5 \mathrm{hmC})$ is a recently identified epigenetic mark which impacts a broad range of biological processes ranging from development to pathogenesis $[9,10] .5 \mathrm{hmC}$ is generated from $5 \mathrm{mC}$ by the ten-eleven translocation (TET) family dioxygenases [11]. Compared to the repressive effect of $5 \mathrm{mC}, 5 \mathrm{hmC}$ is generally believed to have a permissive effect on gene expression [12-15]. Unlike $5 \mathrm{mC}$ which is uniformly dis- 
tributed among different tissues in terms of total mass, $5 \mathrm{hmC}$ displays a tissue-specific mass distribution [16, 17] and low levels of $5 \mathrm{hmC}$ are also frequently observed in many solid tumors compared to corresponding normal tissues [18]. These characteristics suggest that $5 \mathrm{hmC}$ may have potential value in cancer diagnostics [10]. However, in contrast to the intensive studies on cell-free $5 \mathrm{mC}$, cell-free $5 \mathrm{hmC}$ has remained unexploited, partly due to the low levels of $5 \mathrm{hmC}$ in the human genome (10-to 100 -fold $<5 \mathrm{mC}$ ) [17] and the lack of a sensitive low-input $5 \mathrm{hmC}$ DNA sequencing method that would work with the minute amounts of cfDNA available (typically only a few nanograms per $\mathrm{ml}$ of plasma). In this work, we developed a sensitive chemical labeling-based low-input whole-genome $5 \mathrm{hmC}$ sequencing method that allows rapid and reliable sequencing of $5 \mathrm{hmC}$ in cfDNA, and showed that cell-free $5 \mathrm{hmC}$ display distinct features in several types of cancer, which can potentially be used not only to identify cancer types but also to track tumor stage in some cancers.

\section{Results}

\section{Development of cell-free $5 \mathrm{hmC}$ sequencing}

We developed a low-input whole-genome cell-free $5 \mathrm{hmC}$ sequencing method based on selective chemical labeling (hMe-Seal) [13]. hMe-Seal is a robust method that uses $\beta$-glucosyltransferase ( $\beta \mathrm{GT})$ to selectively label $5 \mathrm{hmC}$ with a biotin via an azide-modified glucose for pull-down of 5hmC-containing DNA fragments for sequencing [13] (Supplementary information, Figure S1A). Standard hMe-Seal procedure requires micrograms of DNA. In our modified approach, cfDNA is first ligated with sequencing adapters and $5 \mathrm{hmC}$ is selectively labeled with a biotin group. After capturing cfDNA containing $5 \mathrm{hmC}$ using streptavidin beads, the final library is completed by PCR directly from the beads instead of eluting the captured DNA to minimize sample loss during purification steps (Figure 1A). With this approach we can sequence cell-free $5 \mathrm{hmC}$ readily from 1-10 $\mathrm{ng}$ of cfDNA. By utilizing a pool of $180 \mathrm{bp}$ amplicons bearing C, $5 \mathrm{mC}$ or $5 \mathrm{hmC}$ spiked into cfDNA, we demonstrated that only $5 \mathrm{hmC}$-containing DNA can be detected by PCR from the beads after pull-down (Supplementary information, Figure S1B). This result was confirmed in the final sequencing libraries, which showed over 100-fold enrichment in reads mapping to $5 \mathrm{hmC}$ spike-in DNA (Figure 1B). Furthermore, our approach performed equally well with cfDNA and bulk genomic DNA $(1 \mu \mathrm{g}$ whole blood genomic DNA (gDNA)) (Figure 1B). The final cell-free $5 \mathrm{hmC}$ libraries are highly complex with a median unique nonduplicate map rate of 0.75 when lightly sequenced (median 15 million reads, $\sim 0.5$ fold human genome coverage) (Supplementary information, Figure S1C, S1D and Table S1), and yet technical replicates are highly reproducible (Supplementary information, Figure S1E). We identified $5 \mathrm{hmC}$-enriched regions (hMRs) in the sequence data using a Poisson-based method [19]. hMRs are highly concordant between technical replicates and a pooled sample: over $75 \%$ of hMRs in the pooled sample are in common with each of the replicates (Supplementary information, Figure S1F), reaching the ENCODE standard for ChIP-Seq [20]. These results demonstrate that cell-free $5 \mathrm{hmC}$ can be readily and reliably profiled by the modified hMe-Seal method.

\section{Genome-wide mapping of $5 \mathrm{hmC}$ in cfDNA}

We first sequenced cell-free $5 \mathrm{hmC}$ from eight healthy individuals (Supplementary information, Tables S1 and $\mathrm{S} 2$ ). We also sequenced $5 \mathrm{hmC}$ from whole blood gDNA from two of the individuals as blood is the major contributor to cell-free nucleic acids. Genome-scale profiles showed that the cell-free $5 \mathrm{hmC}$ distributions are nearly identical between healthy individuals and are clearly distinguishable from both the whole blood $5 \mathrm{hmC}$ distribution and the input cfDNA (Supplementary information, Figure S2A). Previous studies of $5 \mathrm{hmC}$ in mouse and human tissues showed that the majority of $5 \mathrm{hmC}$ resides in the gene bodies and promoter proximal regions of the genome $[12,14]$. Genome-wide analysis of hMRs in our cfDNA data showed that the majority $(80 \%)$ are intragenic with most enrichment in exons (observed to expected, $\mathrm{o} / \mathrm{e}=7.29$ ), and depletion in intergenic regions $(\mathrm{o} / \mathrm{e}=0.46)$, consistent with that in whole blood (Supplementary information, Figure S2B and S2C) and in other tissues $[12,14]$. The enrichment of $5 \mathrm{hmC}$ in gene bodies is known to be correlated with transcriptional activity in tissues such as the brain and liver [12-14]. To determine whether this relationship holds in cfDNA, we performed sequencing of the cell-free RNA from the same individual [21]. By dividing genes into three groups according to their cell-free RNA expression and plotting the average cell-free $5 \mathrm{hmC}$ profile along gene bodies (metagene analysis), we discovered an enrichment of $5 \mathrm{hmC}$ in and around gene bodies of more highly expressed genes (Figure $1 \mathrm{C}$ ). These results demonstrate that cell-free $5 \mathrm{hmC}$ is derived from various tissue types and contains information from tissues other than the blood.

Since cell-free $5 \mathrm{hmC}$ were mostly enriched in the intragenic regions, we next used genic $5 \mathrm{hmC}$ fragments per kilobase of gene per million mapped reads (FPKM) to further compare the cell-free hydroxymethylome with the whole blood hydroxymethylome. Indeed, unbiased analysis of genic $5 \mathrm{hmC}$ using $t$-distributed stochastic 
A

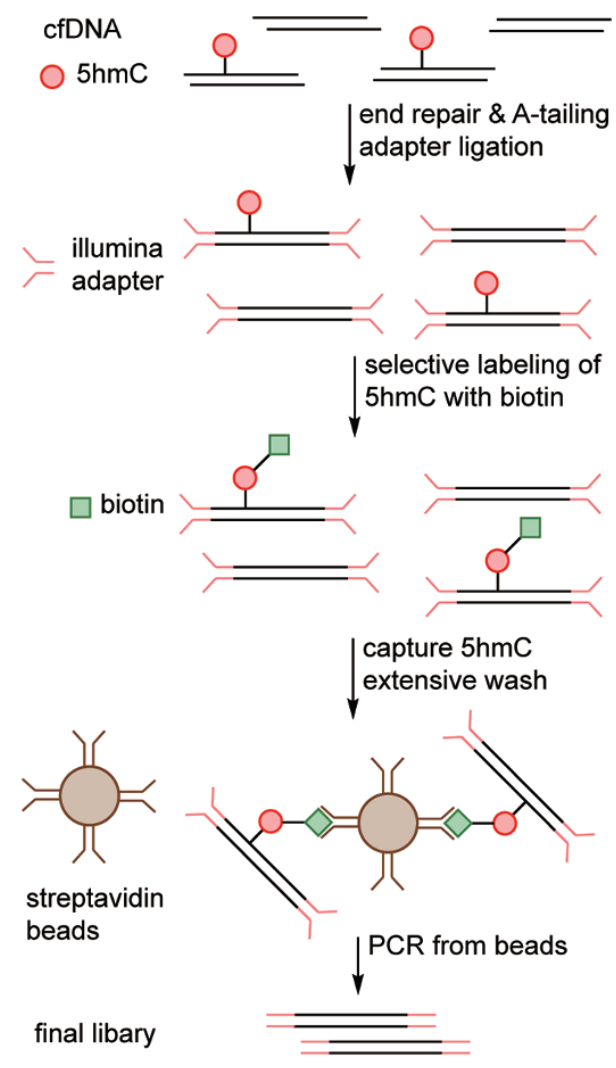

B

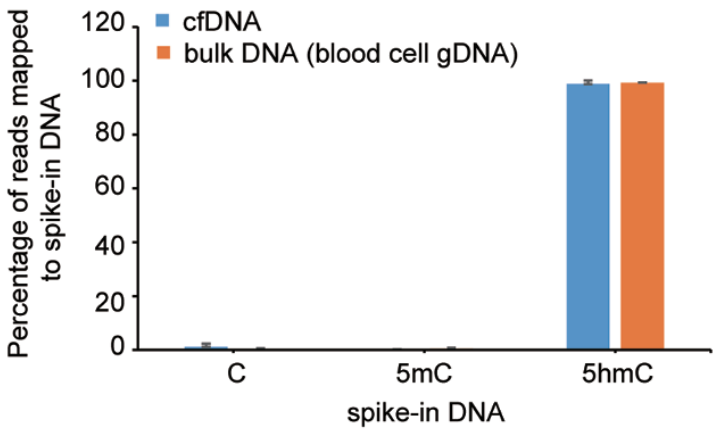

C

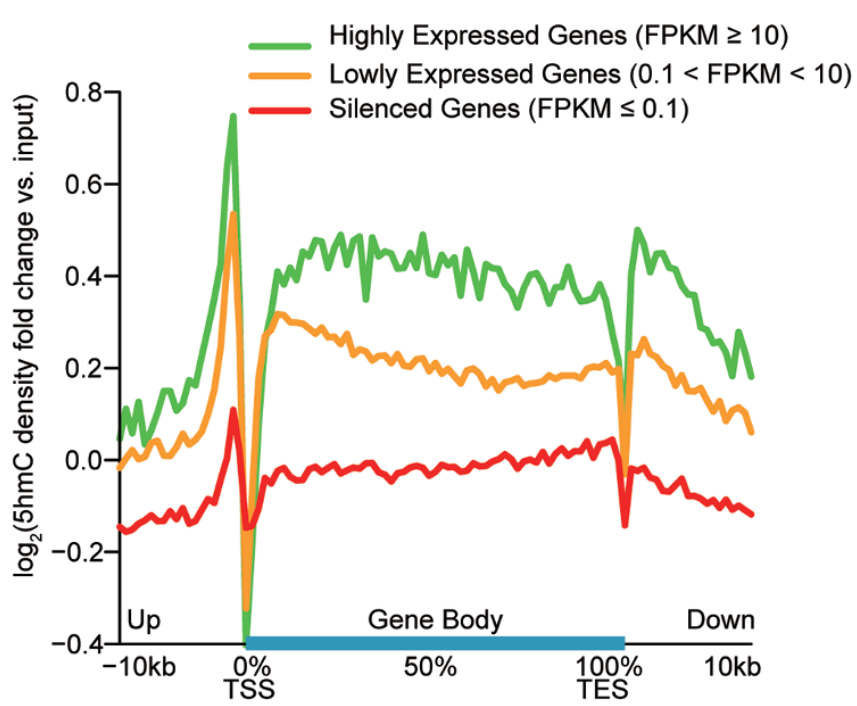

Figure 1 Sequencing of $5 \mathrm{hmC}$ in cfDNA. (A) General procedure of cell-free $5 \mathrm{hmC}$ sequencing. cfDNA is ligated with Illumina adapter and labeled with biotin on $5 \mathrm{hmC}$ for pull-down with streptavidin beads. The final library is completed by directly PCR from streptavidin beads. (B) Percentage of reads mapped to spike-in DNA in the sequencing libraries. Error bars indicate SD. (C) Metagene profiles of $\log _{2}$ fold change of cell-free $5 \mathrm{hmC}$ to input cfDNA ratio in genes ranked according to their expression in cell-free RNA-Seq.

neighbor embedding ( $t \mathrm{SNE}$ ) [22] showed strong separation between the cell-free and whole blood samples (Supplementary information, Figure S2D). We used the limma package [23] to identify 2082 differentially hydroxymethylated genes between whole blood and cellfree samples ( $q$-values (Benjamini and Hochberg adjusted $P$-values $)<0.01$, fold change $>2$, Supplementary information, Figure S3A). Notably, the 735 blood-specific $5 \mathrm{hmC}$ enriched genes showed increased expression in whole blood compared to the 1347 cell-free-specific $5 \mathrm{hmC}$ enriched genes [24] ( $P$-value $<2.2 \times 10^{-16}$, Welch $t$-test) (Supplementary information, Figure S3B). In agreement with the differential expression, gene ontology (GO) analysis [25] of blood-specific 5hmC enriched genes mainly identified blood cell-related processes (Supplementary information, Figure S3C), whereas cell-free-specific $5 \mathrm{hmC}$ enriched genes identified much more diverse biological processes (Supplementary information, Figure S3D). Examples of whole blood-specific (FPR1, FPR2) and cell-free-specific (GLP1R) 5hmC enriched genes are shown in Supplementary information, Figure S3E. Together, these results provide further evidence that a variety of tissues contribute $5 \mathrm{hmC}$ to cfDNA and that measurement of this is a rough proxy for gene expression.

Stage-dependent loss of $5 \mathrm{hm} C$ in lung cancer cfDNA

To explore the diagnostic potential of cell-free $5 \mathrm{hmC}$, we applied our method to sequence cfDNA of a panel of 49 treatment-naïve primary cancer patients, including 15 lung cancer, 10 hepatocellular carcinoma (HCC), 7 pancreatic cancer, 4 glioblastoma (GBM), 5 gastric can- 
cer, 4 colorectal cancer, 4 breast cancer patients (Supplementary information, Tables S3-S9). These patients vary from early stage cancer to late stage metastatic cancer. In lung cancer, we observed a progressive global loss of $5 \mathrm{hmC}$ enrichment from early stage non-metastatic lung cancer to late stage metastatic lung cancer compared to healthy cfDNA, and it gradually resembled that of the unenriched input cfDNA (Figure 2A). Unbiased gene body analysis using $t$ SNE also showed a stage-dependent migration of the lung cancer profile from the healthy profile to one resembling the unenriched input cfDNA
(Supplementary information, Figure S4A). Notably, even the early stage lung cancer samples are highly separated from the healthy samples (Supplementary information, Figure S4A). We further confirmed the global hypohydroxymethylome events using other metrics. First, most differential genes in metastatic lung cancer ( $q$-values $<$ 1e-7, 1159 genes) showed stage-dependent depletion of $5 \mathrm{hmC}$ compared to healthy samples (Figure 2B). Second, the metagene profile showed a stage-dependent depletion of gene body $5 \mathrm{hmC}$ signal and resemblance of the unenriched input cfDNA (Supplementary information, Figure

A

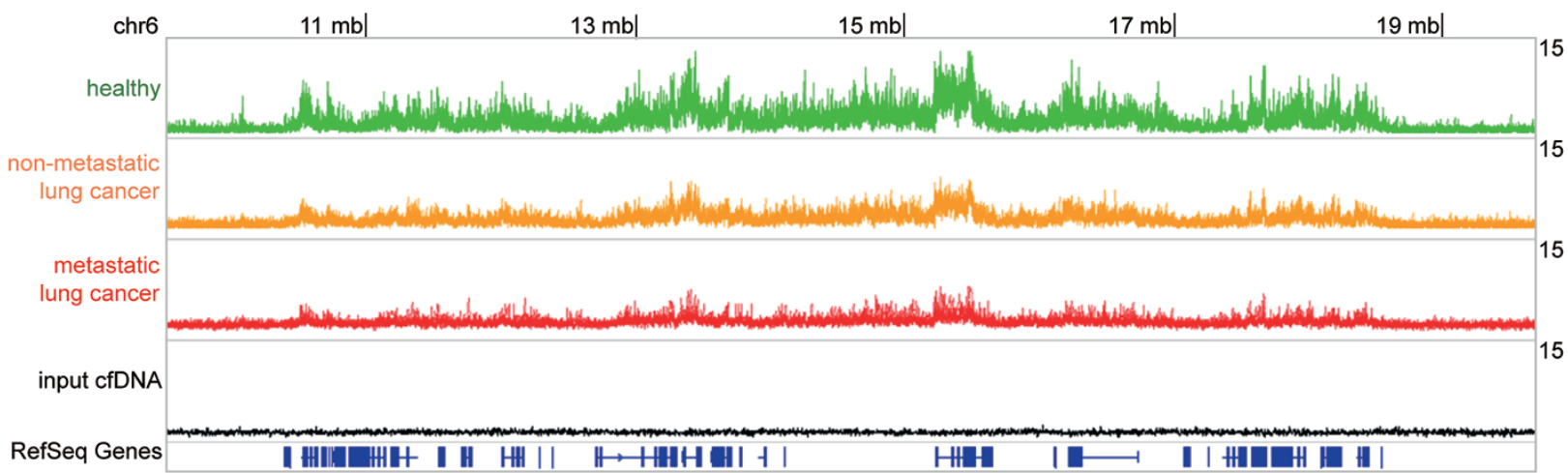

B

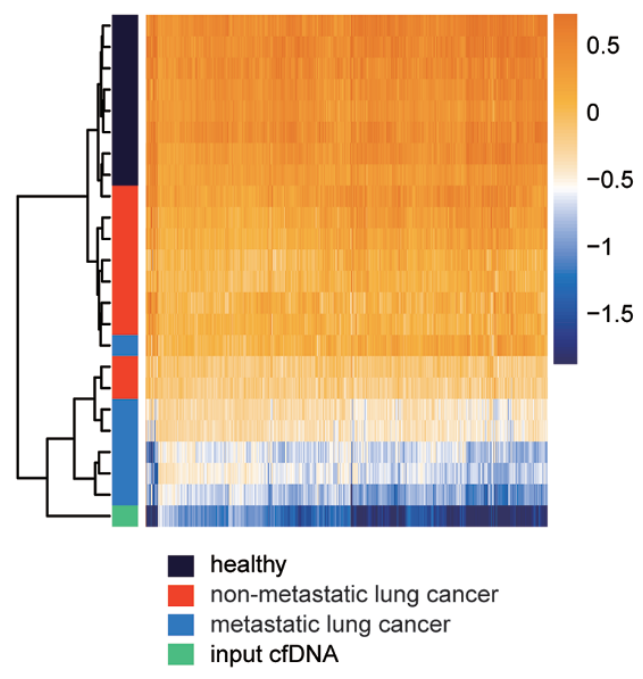

C

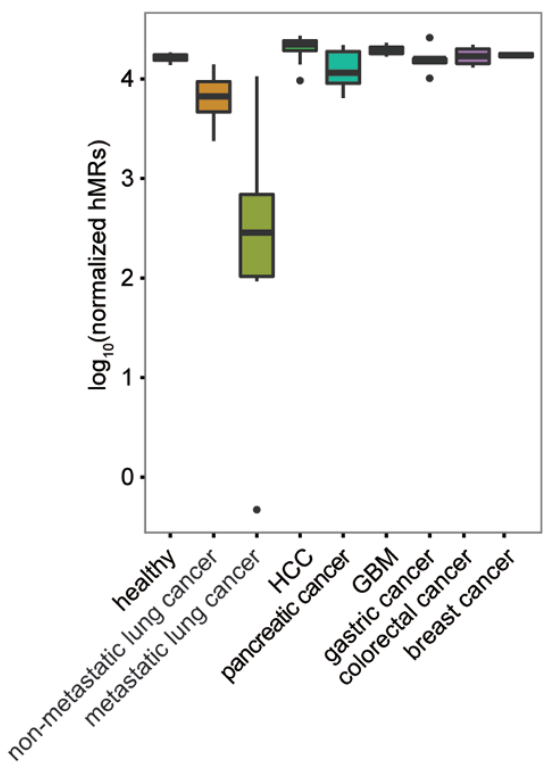

D

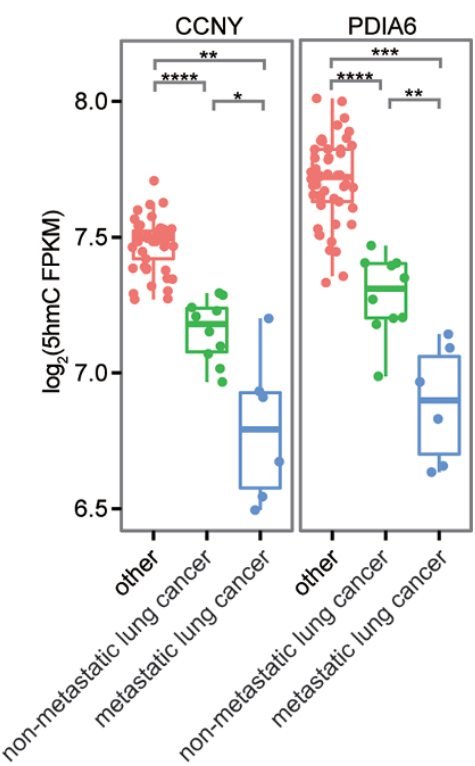

Figure 2 Lung cancer leads to progressive loss of $5 \mathrm{hmC}$ enrichment in cfDNA. (A) Genome browser view of the cell-free $5 \mathrm{hmC}$ distribution in a $10 \mathrm{mb}$ region in chromosome 6 . The overlapping tracks of healthy, non-metastatic lung cancer, metastatic lung cancer and input cfDNA samples are shown in line plot. (B) Heatmap of 1159 metastatic lung cancer differential genes in healthy, lung cancer samples and the unenriched input cfDNA. Hierarchical clustering was performed across genes and samples. (C) Boxplot of number of hMRs (normalized to 1 million reads) identified in each group. (D) Boxplots of CCNY and PDIA6 5hmC FPKM in lung cancer and other cfDNA samples. ${ }^{\star} P<0.05,{ }^{* *} P<0.01$, ${ }^{* * *} P<0.001$, ${ }^{* \star * *} P<1 \mathrm{e}-5$, Welch $t$-test. 
S4B). Third, there is a dramatic decrease in the number of hMRs identified in lung cancer, especially in metastatic lung cancer compared to healthy and other cancer samples (Figure 2C). These data collectively indicate stage-dependent global loss of $5 \mathrm{hmC}$ levels in lung cancer cfDNA.

It should be noted that the global loss of $5 \mathrm{hmC}$ enrichment seen in lung cancer cfDNA is not due to the failure of our enrichment method, as the spike-in control in all samples including the lung cancer samples showed high enrichment of $5 \mathrm{hmC}$-containing DNA (Supplementary information, Figure S4C). It is also a phenomenon unique to lung cancer that is not observed in other cancers we tested, evidenced by the number of hMRs (Figure 2C) and the metagene profiles (Supplementary information, Figure S4B). Examples of $5 \mathrm{hmC}$ depleted genes in lung cancer are shown in Figure 2D and Supplementary information, Figure S4D. Lung cancer tissue is known to have a low level of $5 \mathrm{hmC}$ compared to normal lung tissue [18], and lung has a relatively large contribution to cfDNA [21]. It is plausible that lung cancer, especially metastatic lung cancer, causes large quantities of hypohydroxymethylated gDNA to be released into cfDNA, effectively diluting the cfDNA and leading to the depletion of $5 \mathrm{hmC}$ in the cell-free $5 \mathrm{hmC}$ landscape. Alternatively or in combination, the cfDNA hypohydroxymethylation could originate from blood gDNA hypohydroxymethylation observed in metastatic lung cancer patients as recently reported [26]. Taken together these results indicate that cell-free $5 \mathrm{hmC}$ sequencing may potentially serve as a powerful tool for early lung cancer detection as well as monitoring lung cancer progression and metastasis.

\section{Monitoring treatment and recurrence in HCC}

For HCC, we also sequenced cell-free $5 \mathrm{hmC}$ from seven patients with hepatitis B (HBV) infection, since most HCC cases are secondary to viral hepatitis infections (Supplementary information, Table S4). Unbiased gene level analysis by $t \mathrm{SNE}$ revealed that HCC patients in general can be separated from the HBV-infected patients and healthy individuals based on the cell-free $5 \mathrm{hmC}$ patterns (Figure 3A). HCC-specific differential genes ( $q$-values $<0.001$, fold change $>1.41,1006$ genes) could separate $\mathrm{HCC}$ from healthy and most of the HBV samples (Figure 3B). Both HCC specifically enriched and depleted genes can be identified compared to other cfDNA samples (Figure 3B), and the enriched genes (379 genes) showed increased expression in liver tissue compared to the depleted genes (637 genes) [24] ( $P$-values $<2.2 \times$ $10^{-16}$, Welch $t$-test) (Supplementary information, Figure $\mathrm{S} 5 \mathrm{~A}$ ), consistent with the permissive effect of $5 \mathrm{hmC}$ on gene expression. An example of HCC-specific $5 \mathrm{hmC}$ en- riched genes is AHSG, a secreted protein highly expressed in the liver [24] (Figure 3C; Supplementary information, Figure S5B and S5C), and an example of HCC-specific $5 \mathrm{hmC}$ depleted genes is TET2, one of the enzymes that generate $5 \mathrm{hmC}$ and a tumor suppressor down-regulated in HCC [27] (Figure 3D; Supplementary information, Figure S5D). Together, these results point to a model where virus infection and $\mathrm{HCC}$ development lead to a gradual damage of liver tissue and increased presentation of liver DNA in the blood.

To further explore the potential of cell-free $5 \mathrm{hmC}$ for monitoring treatment and disease progression, we followed four of the HCC patients who underwent surgical resection, out of which three of them had recurrent disease (Supplementary information, Table S4). Analysis of serial plasma samples from these patients (pre-operation/preop; post-operation/post-op; and recurrence) with $t \mathrm{SNE}$ revealed that post-op samples clustered with healthy samples, whereas the recurrence samples clustered with HCC (Figure 3E). This pattern was also reflected by changes in the $5 \mathrm{hmC}$ FPKM of AHSG and TET2 (Figure 3C and $3 \mathrm{D})$. As an example of using cell-free $5 \mathrm{hmC}$ for tracking HCC treatment and progression, we employed linear discriminant analysis (LDA) to define a linear combination of the HCC-specific differential genes (Figure 3D) into to a single value (the HCC score) that best separated the preop HCC samples from the healthy and HBV samples. We then calculated the HCC score for the post-op and recurrence HCC samples, and showed that the HCC score can accurately track the treatment and recurrence states (Supplementary information, Figure S5E). Together, these results indicate that cell-free $5 \mathrm{hmC}$ sequencing presents an opportunity to detect HCC, as well as monitor treatment outcome and disease recurrence.

\section{Pancreatic cancer impacts the cell-free $5 \mathrm{hmC}$}

We also found pancreatic cancer produced drastic changes in its cell-free hydroxymethylome, even in some early stage pancreatic cancer patients (Supplementary information, Table S5). Like HCC, pancreatic cancer lead to both up-regulated and down-regulated $5 \mathrm{hmC}$ genes compared to healthy individuals ( $q$-value $<0.01$, fold change $>2,713$ genes) (Supplementary information, Figure S6A). Examples of pancreatic cancer-specific 5hmC-enriched and depleted genes compared to other cfDNA samples are shown in Supplementary information, Figure S6B-S6E. Our results suggest that cell-free $5 \mathrm{hmC}$ sequencing can be potentially valuable for early detection of pancreatic cancer.

\section{Copy number variation estimation}

Copy number variation (CNV) can be detected from 
A

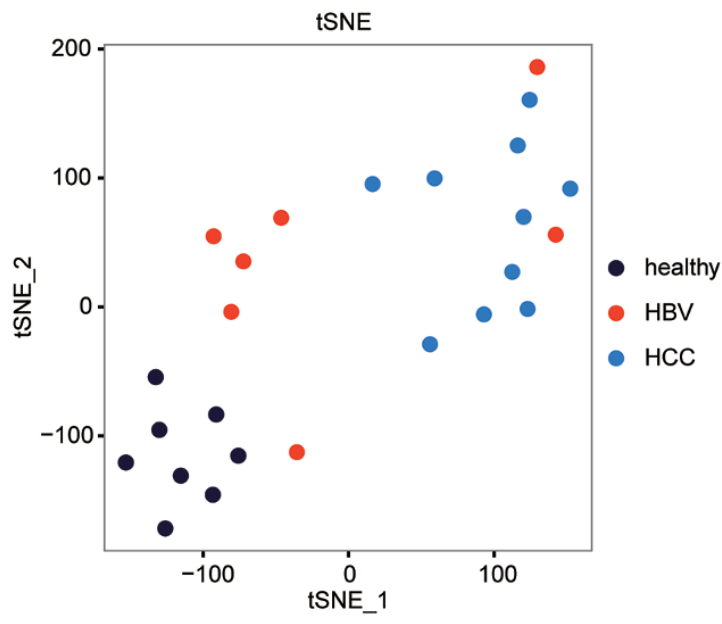

C

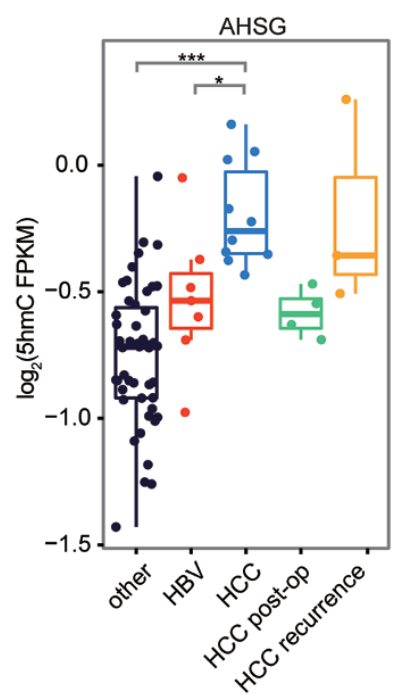

B

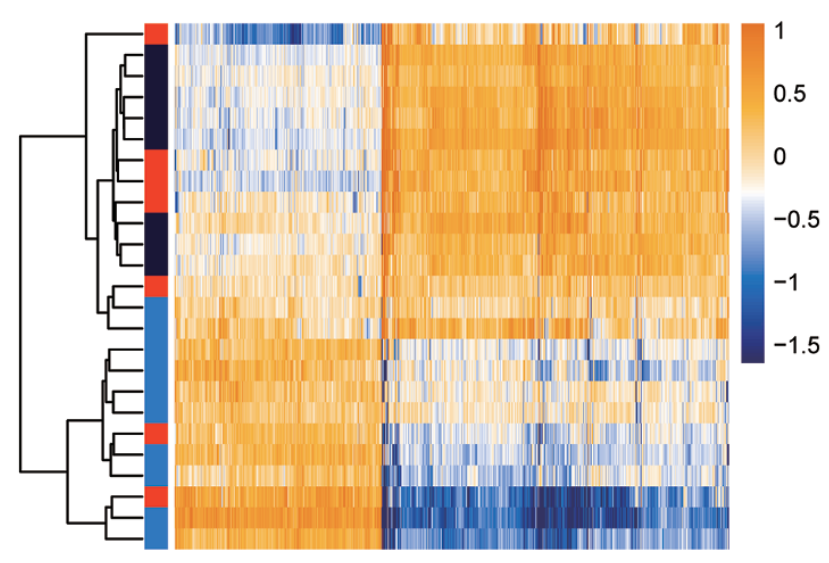

E

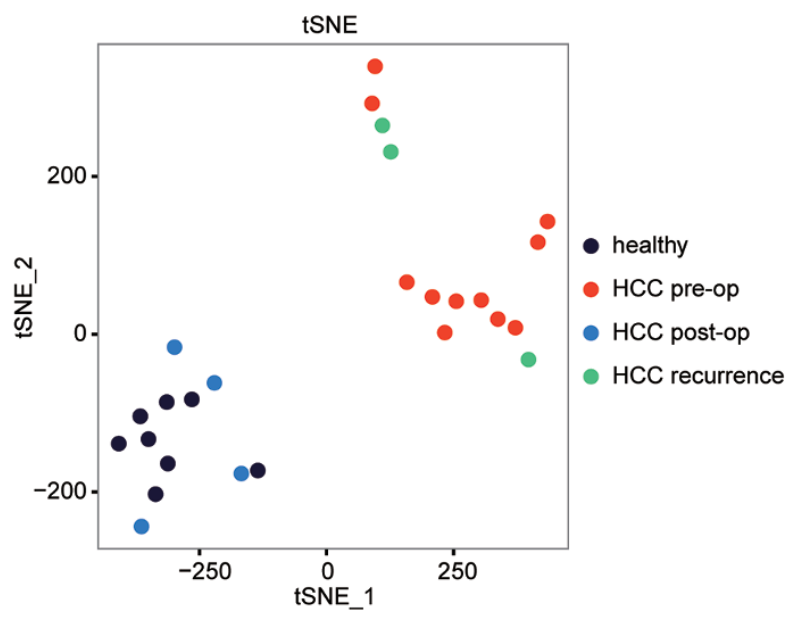

Figure 3 Cell-free $5 \mathrm{hmC}$ for monitoring HCC progression and treatment. (A) tSNE plot of 5hmC FPKM from healthy, HBV and HCC samples. (B) Heatmap of $1006 \mathrm{HCC}$ differential genes in healthy, HBV and HCC samples. Hierarchical clustering was performed across genes and samples. (C, D) Boxplots of AHSG (C) and TET2 (D) 5hmC FPKM in HBV, HCC (pre-op), HCC post-op, HCC recurrence and other cfDNA samples. ${ }^{*} P<0.05$, ${ }^{* *} P<1 \mathrm{e}-4$, ${ }^{* * *} P<1 \mathrm{e}-5$, Welch $t$-test. (E) tSNE plot of $5 \mathrm{hmC}$ FPKM from healthy, HCC pre-op, HCC post-op and HCC recurrence samples.

cfDNA sequencing, mostly in advanced cancer patients, which provides a way to assess the tumor burden in the cfDNA [3]. To assess the tumor burden in our samples and to explore the relation between $\mathrm{CNV}$ contained from unenriched input cfDNA sequencing and the $5 \mathrm{hmC}$ enrichment sequencing, we also sequenced the input cfDNA in 47 samples (Supplementary information, Table $\mathrm{S} 10)$. We analyzed the CNV from these input cfDNA sequencing with $1 \mathrm{mb}$ bin [28], and as expected we could detect large-scale CNV from about $20 \%$ of the cancer samples, mostly in late stage cancer samples (Supple- mentary information, Figure S7A). We then analyzed the $\mathrm{CNV}$ from the corresponding $5 \mathrm{hmC}$ enrichment sequencing and interestingly, we found matched CNV patterns in several cases (Supplementary information, Figure S7A). For example we could detect chromosome wise CNV in lung293 and lung417, two metastatic lung cancer samples, from input cfDNA sequencing (Supplementary information, Figure S7B and S7C). These samples displayed large-scale cell-free $5 \mathrm{hmC}$ changes and correspondingly, the $\mathrm{CNV}$ patterns detected from the $5 \mathrm{hmC}$ enrichment sequencing mimic the CNV patterns detected 
from input cfDNA sequencing (Supplementary information, Figure S7D and S7E). This result supports the notion that $5 \mathrm{hmC}$ enriched cfDNA contains significant portion of tumor-derived cfDNA and therefore represents $5 \mathrm{hmC}$ patterns in tumor cells. It also shows that $5 \mathrm{hmC}$ sequencing and $\mathrm{CNV}$ analysis could complement each other in circulating tumor DNA analysis.

\section{Cancer type and stage prediction}

Although there has been great interest in using cfDNA as a "liquid biopsy" for cancer detection, it has been challenging to identify the origin of tumor cfDNA and hence the location of the tumor. We discovered from $t \mathrm{SNE}$ analysis of all seven cancer types that lung cancer, $\mathrm{HCC}$ and pancreatic cancer showed distinct signatures and could be readily separated from each other and healthy samples (Figure 4A). The other four types of cancer displayed relatively minor changes compared to the healthy samples. Using other features such as the promotor region ( $5 \mathrm{~kb}$ upstream of the transcription start site) showed similar patterns (Supplementary information, Figure S8A). We note that no particular cancer type we tested resembled the whole blood profile (Supplementary information, Figure S8B), suggesting that the blood cell contamination is not a significant source of variation. All patients in our panel fall in the same age range as the healthy individuals (Supplementary information, Figure S8C and Tables S2-S9), therefore age is unlikely to be a confounding factor. We also did not observe any batch effect (Supplementary information, Figure S8D).
A

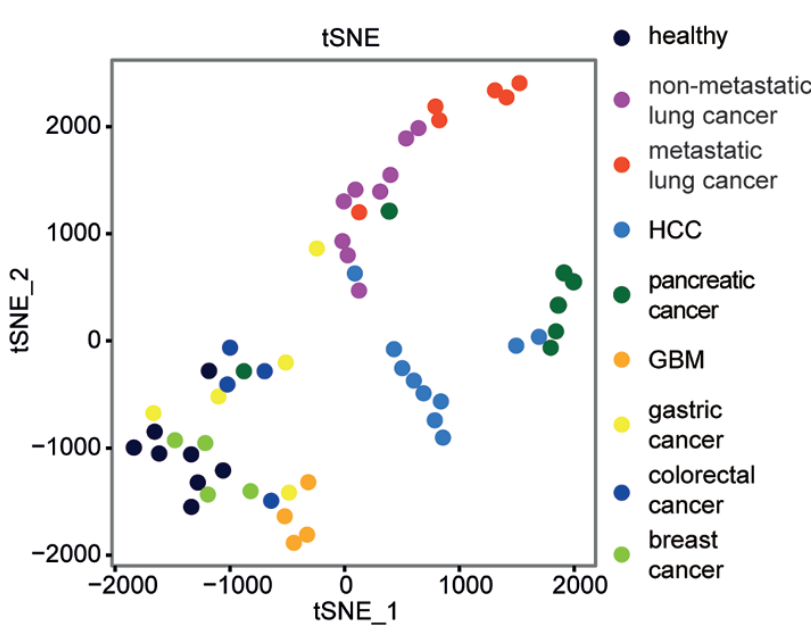

C

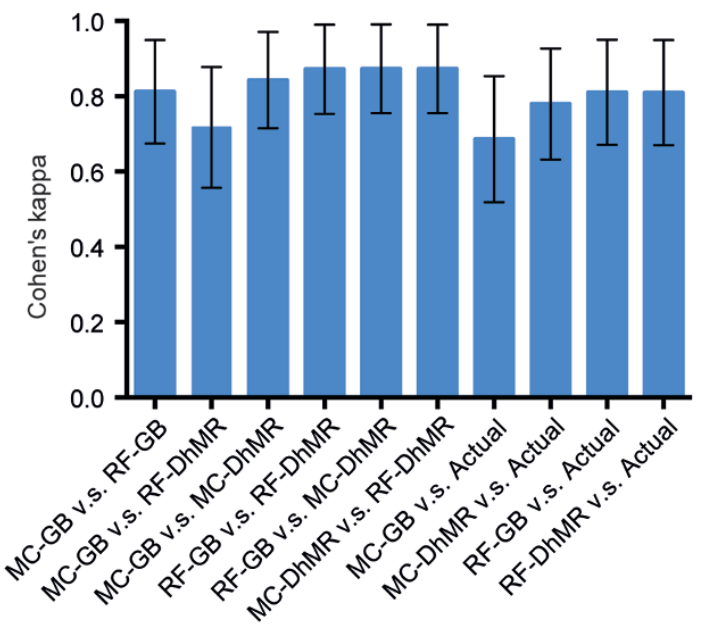

B

\begin{tabular}{|c|c|c|c|c|c|c|c|c|c|c|c|c|c|c|c|c|c|c|c|c|}
\hline \multirow{3}{*}{$\begin{array}{c}\text { Actual } \\
\text { Featureset } \\
\text { Classifier }\end{array}$} & \multicolumn{4}{|c|}{$\begin{array}{l}\text { pancreatic } \\
\text { cancer }\end{array}$} & \multicolumn{4}{|c|}{$\begin{array}{l}\text { non-metastatic } \\
\text { lung cancer }\end{array}$} & \multicolumn{4}{|c|}{$\begin{array}{l}\text { metastatic } \\
\text { lung cancer }\end{array}$} & \multicolumn{4}{|c|}{ HCC } & \multicolumn{4}{|c|}{ healthy } \\
\hline & \multicolumn{2}{|c|}{ Genebody } & \multicolumn{2}{|c|}{ DhMR } & \multicolumn{2}{|c|}{ Genebody } & \multicolumn{2}{|c|}{ DhMR } & \multicolumn{2}{|c|}{ Genebody } & \multicolumn{2}{|c|}{ DhMR } & \multicolumn{2}{|c|}{ Genebody } & \multicolumn{2}{|c|}{ DhMR } & \multicolumn{2}{|c|}{ Genebody } & \multicolumn{2}{|c|}{ DhMR } \\
\hline & $\mathrm{MC}$ & $\mathrm{RF}$ & $\mathrm{MC}$ & $\mathrm{RF}$ & $\mathrm{MC}$ & $\mathrm{RF}$ & MC & $\mathrm{RF}$ & MC & $\mathrm{RF}$ & $\mathrm{MC}$ & $\mathrm{RF}$ & $\mathrm{MC}$ & $\mathrm{RF}$ & $\mathrm{MC}$ & $\mathrm{RF}$ & MC & $\mathrm{RF}$ & $\mathrm{MC}$ & RF \\
\hline $\begin{array}{l}\text { pancreatic } \\
\text { cancer }\end{array}$ & 4 & 5 & 5 & 5 & 1 & 1 & 1 & 0 & 0 & 0 & 0 & 0 & 1 & 0 & 0 & 1 & 1 & 1 & 1 & 1 \\
\hline $\begin{array}{l}\text { non-metastatic } \\
\text { lung cancer }\end{array}$ & 1 & 0 & 1 & 0 & 6 & 8 & 7 & 8 & 2 & 0 & 1 & 0 & 0 & 1 & 0 & 1 & 0 & 0 & 0 & 0 \\
\hline $\begin{array}{c}\text { metastatic } \\
\text { lung cancer }\end{array}$ & 0 & 0 & 0 & 0 & 1 & 1 & 2 & 2 & 5 & 5 & 4 & 4 & 0 & 0 & 0 & 0 & 0 & 0 & 0 & 0 \\
\hline $\mathrm{HCC}$ & 2 & 1 & 1 & 1 & 0 & 1 & 0 & 0 & 0 & 0 & 0 & 0 & 8 & 8 & 9 & 9 & 0 & 0 & 0 & 0 \\
\hline healthy & 1 & 0 & 0 & 0 & 0 & 0 & 0 & 0 & 0 & 0 & 0 & 0 & 0 & 0 & 0 & 0 & 7 & 8 & 8 & 8 \\
\hline
\end{tabular}

Figure 4 Cancer type and stage prediction with cell-free $5 \mathrm{hmC}$. (A) tSNE plot of 5hmC FPKM in cfDNA from healthy and various cancer samples. (B) The actual and predicted classification by leave-one-out cross-validation using Mclust (MC) and Random Forest (RF) algorithm, based on two feature sets (gene body and DhMR). (C) The Cohen's kappa coefficient for measuring inter-classifier agreement (GB for gene body). The error bar indicates 95\% confidence interval of the Cohen's kappa estimate. 
To further demonstrate the potential of cfDNA $5 \mathrm{hmC}$ as a biomarker to predict cancer types, we employed two widely used machine learning methods, the Gaussian mixture model [29] and Random Forest [30]. We focused on the prediction of HCC, pancreatic cancer, non-metastatic and metastatic lung cancer. On the basis of three rules (see Materials and Methods section), we identified genes (Supplementary information, Table S11) whose average gene body $5 \mathrm{hmC}$ levels could either distinguish cancer groups from healthy groups or between cancer groups. In addition to using gene body data, the $5 \mathrm{hmC}$ on non-coding regions could also potentially serve as a biomarker in predicting cancer types [9]. We therefore designed another set of features by investigating each of the $2 \mathrm{~kb}$ windows of the entire genome and identified differential hMRs (DhMRs) for each cancer type (see Materials and Methods section; Supplementary information, Table S12). We trained the two machine learning algorithms using either differential $5 \mathrm{hmC}$ genes or DhMRs as features and evaluated the leave-one-out (LOO) cross-validation prediction accuracy. The Gaussian mixture model-based predictor (Mclust) had overall successful prediction rates of $75 \%$ and $82.5 \%$, when using gene body and DhMRs as features, respectively (Figure 4B; Supplementary information, Figure S9A and S9B). Mclust-based dimensional reduction showed clear boundaries between the groups (Supplementary information, Figure S9C). When only the type of the cancer is considered, Mclust predictors had higher success rate of $82.5 \%$ and $90 \%$ when using these two feature sets. The Random Forest predictor achieved LOO cross-validation prediction accuracy of $85 \%$, when using either gene body or DhMRs as features (Figure 4B). When only cancer type is considered, Random Forest predictor achieved $87.5 \%$ and $90 \%$ prediction accuracy, with gene body and DhMRs as features, respectively. Distinct $5 \mathrm{hmC}$ profiles in different cancer types of several DhMRs with high variable importance to random forest prediction model could be observed (Supplementary information, Figures S9D, S9E and S10). Finally, we used Cohen's kappa to evaluate the concordance rate between different prediction models [31]. All combinations showed high agreement (Cohen's kappa $\sim 0.8$ ) in inter-classifier comparison and when comparing with the actual classification (Figure 4C). These results support the prospects of using cellfree $5 \mathrm{hmC}$ for cancer diagnostics and staging.

\section{Discussion}

Recent studies have reported that $5 \mathrm{hmC}$ is an important component of the mammalian genome [9, 32]. In this study, we reported an improved hMe-Seal [13] approach to sequence the low levels of $5 \mathrm{hmC}$ in cfDNA, which offers several notable advantages. First, unlike traditional bisulfite sequencing used for cell-free $5 \mathrm{mC}$ sequencing, our method does not further degrade the highly fragmented cfDNA. Second, compared to whole genome approaches including mutational sequencing and bisulfite sequencing, the enrichment for $5 \mathrm{hmC}$ not only enables cost-effective sequencing (10-20 million reads, $\sim 0.5$-fold human genome coverage), but more importantly allows the low-frequency tissue contribution of $5 \mathrm{hmC}$ in cfDNA to be amplified from the dominant blood cell contribution in cfDNA.

We sequenced cell-free $5 \mathrm{hmC}$ from a panel of seven cancer types and focused our analysis on lung cancer, $\mathrm{HCC}$ and pancreatic cancer, the three cancers which displayed the most dramatic impact on the cell-free hydroxymethylome, even in the early stages. Lung and liver are reported to have relatively large contribution to cfDNA [5, 21], and pancreatic cancer is known to invade progressively to the lymph nodes and liver during early stages without remarkable symptoms, which may explain their large impact on the cell-free hydroxymethylome. In lung cancer, we observed a characteristic stage-dependent global loss of cell-free $5 \mathrm{hmC}$ enrichment, whereas in $\mathrm{HCC}$ and pancreatic cancer, we identified significant finer scale changes of cell-free $5 \mathrm{hmC}$ (i.e., gene body and DhMR). In HCC, we also conducted an exploratory study of longitudinal samples whose results suggest that cell-free $5 \mathrm{hmC}$ may be used to monitor treatment and recurrence. Further studies will help elucidate how each cancer causes specific changes in the cell-free hydroxymethylome. Importantly, these three types of cancer displayed distinct patterns in their cell-free hydroxmethylome and we could employ machine learning algorithms trained with cell-free $5 \mathrm{hmC}$ features to predict the three cancer types with high accuracy.

In summary, we report the first proof-of-principle global analysis of hydroxymethylome in cfDNA. Largescale clinical trials are required to fully validate the usefulness and understand potential limitations of this approach. Cell-free $5 \mathrm{hmC}$ contributes a new dimension of information to liquid biopsy-based diagnosis and prognosis; and we anticipate it may become a valuable tool for cancer diagnostics, as well as potentially for other disease areas, including but not limited to neurodegenerative diseases, cardiovascular diseases and diabetes. We envisage this strategy could be readily combined with other genetic and epigenetic-based cfDNA approaches (e.g., CNV analysis as we demonstrated) for increased diagnostic power. Our method represents the first enrichment-based genome-wide approach applied to cfDNA. The general framework of this method can be readily 
adopted to sequence other modifications in cell-free nucleic acids by applying the appropriate labeling chemistry to the modified bases. This would allow a comprehensive and global overview of genetic and epigenetic changes of various disease states, and further increase the power of personalized diagnostics.

\section{Materials and Methods}

\section{Study design}

The overall goal of this study was to explore the diagnostic potential of $5 \mathrm{hmC}$ cfDNA for cancer detection. The objective of the first portion of the study was to determine whether $5 \mathrm{hmC}$ can be sequenced from cfDNA using an enrichment-based method. The objective of the second portion of the study was to determine whether cell-free $5 \mathrm{hmC}$ contains information that can be used for cancer diagnostics. Samples for healthy subjects were obtained from Stanford blood center. HCC and breast cancer patients were recruited in a Stanford University Institutional Review Board-approved protocol. Lung cancer, pancreatic cancer, GBM, gastric cancer and colorectal cancer patients were recruited in a West China Hospital Institutional Review Board-approved protocol. All recruited subjects gave informed consent. No statistical methods were used to predetermine sample size. The experiments were not randomized and the investigators were not blinded to allocation during experiments and outcome assessment. No samples were excluded from the analysis.

\section{Clinical sample collection and processing}

Blood was collected into EDTA-coated Vacutainers. Plasma was collected from the blood samples after centrifugation at $1600 \times g$ for 10 min at $4{ }^{\circ} \mathrm{C}$ and $16000 \times g$ at 10 min at $4{ }^{\circ} \mathrm{C}$. cfDNA was extracted using the Circulating Nucleic Acid Kit (Qiagen). Whole blood genomic DNA was extracted using the DNA Mini Kit (Qiagen) and fragmented using dsDNA Fragmentase (NEB) into average 300 bp. DNA was quantified by Qubit Fluorometer (Life Technologies). Cell-free RNA was extracted using the Plasma/Serum Circulating and Exosomal RNA Purification Kit (Norgen). The extracted cellfree RNA was further digested using Baseline-ZERO DNases (Epicentre) and depleted using Ribo-Zero rRNA Removal Kit (Epicentre) according to a protocol from Clontech.

\section{Spike-in amplicon preparation}

To generate the spiked-in control, lambda DNA was PCR amplified by Taq DNA Polymerase (NEB) and purified by AMPure XP beads (Beckman Coulter) in nonoverlapping 180 bp amplicons, with a cocktail of dATP/dGTP/dTTP and one of the following: dCTP, dmCTP or $10 \%$ dhmCTP (Zymo)/90\% dCTP. Primers sequences are as follows: dCTP FW-5'-CGTTTCCGTTCTTCTTCGTC-3', RV-5'-TACTCGCACCGAAAATGTCA-3'; dmCTP FW-5'-GTGGCGGGTTATGATGAACT-3', RV-5'-CATAAAATGCGGGGATTCAC-3'; 10\% dhmCTP/90\% dCTP FW-5'-TGAAAACGAAAGGGGATACG-3', RV-5'-GTCCAGCTGGGAGTCGATAC-3'.

$5 \mathrm{hm}$ C library construction, labeling, capture and high-throughput sequencing

cfDNA (1-10 ng) or fragmented whole blood genomic DNA $(1 \mu \mathrm{g})$ spiked with amplicons $(0.01 \mathrm{pg}$ of each amplicon per 10 ng DNA) was end repaired, 3'-adenylated and ligated to DNA Barcodes (Bioo Scientific) using KAPA Hyper Prep Kit (Kapa Biosystems) according to the manufacturer's instructions. Ligated DNA was incubated in a $25 \mu$ solution containing $50 \mathrm{mM}$ HEPES buffer ( $\mathrm{pH}$ 8), $25 \mathrm{mM} \mathrm{MgCl} 2,60 \mu \mathrm{M}$ UDP-6- $\mathrm{N}_{3}$-Glc (Active Motif) and $12.5 \mathrm{U} \beta \mathrm{GT}$ (Thermo) for $2 \mathrm{~h}$ at $37{ }^{\circ} \mathrm{C}$. After that, 2.5 $\mu 1$ DBCO-PEG4-biotin (Click Chemistry Tools, $20 \mathrm{mM}$ stock in DMSO) was directly added to the reaction mixture and incubated for $2 \mathrm{~h}$ at $37^{\circ} \mathrm{C}$. Next, $10 \mu \mathrm{g}$ sheared salmon sperm DNA (Life Technologies) was added into the reaction mixture and the DNA was purified by Micro Bio-Spin 30 Column (Bio-Rad). The purified DNA was incubated with $0.5 \mu 1 \mathrm{M} 270$ Streptavidin beads (Life Technologies) pre-blocked with salmon sperm DNA in buffer 1 (5 $\mathrm{mM}$ Tris $\mathrm{pH} 7.5,0.5 \mathrm{mM}$ EDTA, $1 \mathrm{M} \mathrm{NaCl}$ and $0.2 \%$ Tween 20 ) for $30 \mathrm{~min}$. The beads were subsequently undergone three 5 -min washes each with buffer 1 , buffer 2 (buffer 1 without $\mathrm{NaCl}$ ), buffer 3 (buffer 1 with $\mathrm{pH}$ 9) and buffer 4 (buffer 3 without $\mathrm{NaCl}$ ). All binding and washing were done at room temperature with gentle rotation. Beads were then resuspended in water and amplified with 14 (cfDNA) or 9 (whole blood genomic DNA) cycles of PCR amplification using Phusion DNA polymerase (NEB). The PCR products were purified using AMPure XP beads. Separate input libraries were made by direct PCR from ligated DNA without labeling and capture. For technical replicates, cfDNA from the same subject was divided into two technical replicates. Pair-end $75 \mathrm{bp}$ sequencing was performed on the NextSeq instrument.

\section{Data processing and gene body analysis}

FASTQ sequences were aligned to UCSC/hg19 with Bowtie2 v2.2.5 [33] and further filtered with samtools-0.1.19 [34] (parameters used: samtools view -f 2 -F 1548 -q 30 and samtools rmdup) to retain unique non-duplicate matches to the genome. Pair-end reads were extended and converted into bedgraph format normalized to the total number of aligned reads using bedtools [35], and then converted to bigwig format using bedGraphToBigWig from the UCSC Genome Browser for visualization in Integrated Genomics Viewer $[36,37]$. FASTQ sequences were also aligned to the three spike-in control sequences to evaluate the pull-down efficiency. The spike-in control is only used as a validation of successful pull-down in each sample. hMRs were identified with MACS [19] using unenriched input DNA as background and default setting ( $P$-value cutoff $1 \mathrm{e}-5)$. Genomic annotations of hMRs were performed by determining the percentage of hMRs overlapping each genomic regions $\geq 1 \mathrm{bp}$. Metagene profile was generated using ngs.plot [38]. 5hmC FPKM were calculated using the fragment counts in each RefSeq gene body obtained by bedtools. For differential analyses, genes shorter than $1 \mathrm{~kb}$ or mapped to chromosome $\mathrm{X}$ and $\mathrm{Y}$ were excluded. Differential genic $5 \mathrm{hmC}$ analysis was performed using the limma package in R [23]. GO analyses were performed using DAVID Bioinformatics Resources 6.7 with GOTERM_BP_FAT [25, 39]. Tissue-specific gene expression was obtained from BioGPS [24, 40, 41]. For $t$ SNE plot, the Pearson correlation of gene body $5 \mathrm{hmC}$ FPKM was used as the distance matrix to $t$ SNE. MA-plot, hierarchical clustering, $t \mathrm{SNE}$, LDA and heatmaps were done in $\mathrm{R}$.

\section{Cell-free RNA library construction and high-throughput se- quencing}

Cell-free RNA library was prepared using ScriptSeq v2 RNA- 
Seq Library Preparation Kit (Epicentre) following the FFPE RNA protocol with 19 cycles of PCR amplification. The PCR products were then purified using AMPure XP beads. Pair-end 75 bp sequencing was performed on the NextSeq instrument. RNA-seq reads were first trimmed using Trimmomatic-0.33 [42] and then aligned using tophat-2.0.14 [43]. RPKM expression values were extracted using cufflinks-2.2.1 [44] using RefSeq gene models.

\section{CNV estimation}

The hg19 human genome was split into $1 \mathrm{mb}$ bin and bin counts were generated using bedtools intersect. Mappability score of each bin was then assessed by average mappability using mappability track of hg19 from UCSC $(\mathrm{kmer}=75)$. Bins with mappability score under 0.8 were eliminated from further analysis. GC content percentages in each bin were calculated using getGC.hg19 from R package PopSV (1.0.0) and GC bias was corrected by fitting a LOESS model (correct.GC from R package PopSV 1.0.0). The corrected bin counts were then scaled by mean bin count of each sample and centered at 2. For estimation of CNV, we cutoff the corrected bin counts higher than 5 to minimize impact of extreme values. Moving averages with window size of $20 \mathrm{mb}$ were then calculated within each chromosome, as the final estimation of CNV.

\section{Cancer type and stage prediction}

Lung cancer, pancreatic cancer, HCC and healthy samples $(n=$ 40 ) were included in the following analyses and LOO cross-validation was performed. With each iteration of LOO, one sample was left out first, and the remaining 39 samples were used for feature selection and as a training data set. The left out sample was then used to test the prediction accuracy of the machine learning model. Two types of feature selection were performed as independent analysis. In the "gene body" approach, cancer type-specific marker genes were selected by performing a Student's $t$-test between (1) one cancer group and healthy group, (2) one cancer group and other cancer samples, (3) two different cancer groups. Benjamini and Hochberg correction was then performed for the raw $P$-value and the genes were then sorted by $q$-value. The top five genes with smallest $q$-value from each of these comparisons were selected as feature set to train the classifier. The second approach to finding features ("DhMR") attempted to achieve higher resolution by first breaking the reference genome (hg19) into $2 \mathrm{~kb}$ windows in silico and then calculating $5 \mathrm{hmC} \mathrm{FPKM} \mathrm{value} \mathrm{for} \mathrm{each} \mathrm{of} \mathrm{the} \mathrm{window.}$ Blacklisted genomic regions that tend to show artifact signal according to ENCODE were filtered before down-stream analysis [45]. For cancer type-specific DhMRs, Student's $t$-test and Benjamini and Hochberg correction of $P$-values were performed for comparison pairs same as previously performed for identifying cancer-specific genes. The top five DhMRs with smallest $q$-value from each comparison were chosen for each cancer type. Random forest and Gaussian model-based Mclust classifier were performed on the data set using previously described features (gene bodies and DhMRs). Classifiers were trained on lung cancer, pancreatic cancer, HCC and healthy samples. The same random seed (seed = 5) was used in every random forest analysis for consistency. The top 15 features shared by at least 30 LOO iterations with the highest mean decrease Gini with the highest variable importance were plotted. Gaussian mixture model analysis was performed using Mclust R package [29]. For Mclust model-based classifier train- ing, a Bayesian information criterion (BIC) plot was performed for visualization of the classification efficacy of different multivariate mixture models. By default, the EEI model (diagonal, equal volume and shape) or VII model (spherical, unequal volume) with EDDA model-type (single component for each class with the same covariance structure among classes) were chosen for Mclust classification. Cohen's kappa was then calculated for assessment of the interclassifier concordance.

\section{Statistical analysis}

We used unpaired two-tailed $t$-tests (Welch $t$-test) for normally distributed data in which two comparison groups were involved. In the case of multiple comparisons, Benjamini and Hochberg correction was then performed for the raw $P$-value to obtain the $q$-value. Random forest and Gaussian model-based Mclust were used as machine classifiers. Cohen's kappa was used for evaluating the predictive value of cell-free DNA $5 \mathrm{hmC}$ sequencing and interclassifier concordance. $t \mathrm{SNE}$ was used for dimension reduction and visualization. Statistical analyses were performed in R 3.3.2.

All sequencing data were deposited in the Gene Expression Omnibus (http://www.ncbi.nlm.nih.gov/geo) under accession number GSE81314.

\section{Acknowledgments}

We would like to acknowledge N Neff and G Mantalas for high-throughput sequencing; L Penland and J Beausang for sample collection; other members of the Quake and Xie Labs for discussions and support; R Altman and W Zhou for critical reading of the manuscript. This work was supported by the National Natural Science Foundation of China (31571327 and 91631111 to DX), National Institutes of Health (U01 CA154209 to SRQ) and Department of Defense (W81XWH1110287 to SRQ).

\section{Author Contributions}

C-XS, JD, DX and SRQ conceived the study and designed the experiments. C-XS performed the experiments with the help from LM, YC and BD. C-XS analyzed data with help from SY, ZT and DX. LM, AW, YZ, BL, JX, WZ, JH, ZZ, SSJ, M-SC, SS, WL and YW recruited patients, collected blood and organized clinical information. C-XS and SRQ wrote the manuscript with input and comments from SY, DX and M-SC.

\section{Competing Financial Interests}

Stanford has filed patent applications based on this work. C-XS and SRQ are founders of Bluestar Genomics.

\section{References}

1 Fan HC, Blumenfeld YJ, Chitkara U, Hudgins L, Quake SR. Noninvasive diagnosis of fetal aneuploidy by shotgun sequencing DNA from maternal blood. Proc Natl Acad Sci USA 2008; 105:16266-16271.

2 Snyder TM, Khush KK, Valantine HA, Quake SR. Universal noninvasive detection of solid organ transplant rejection. Proc Natl Acad Sci USA 2011; 108:6229-6234.

3 Wan JC, Massie C, Garcia-Corbacho J, et al. Liquid biopsies come of age: towards implementation of circulating tumour 
DNA. Nat Rev Cancer 2017; 17:223-238.

4 Chan KC, Jiang P, Chan CW, et al. Noninvasive detection of cancer-associated genome-wide hypomethylation and copy number aberrations by plasma DNA bisulfite sequencing. Proc Natl Acad Sci USA 2013; 110:18761-18768.

5 Sun K, Jiang P, Chan KC, et al. Plasma DNA tissue mapping by genome-wide methylation sequencing for noninvasive prenatal, cancer, and transplantation assessments. Proc Natl Acad Sci USA 2015; 112:E5503-E5512.

6 Wen L, Li J, Guo H, et al. Genome-scale detection of hypermethylated $\mathrm{CpG}$ islands in circulating cell-free DNA of hepatocellular carcinoma patients. Cell Res 2015; 25:1250-1264.

7 Lehmann-Werman R, Neiman D, Zemmour H, et al. Identification of tissue-specific cell death using methylation patterns of circulating DNA. Proc Natl Acad Sci USA 2016; 113:E1826-E1834.

8 Guo S, Diep D, Plongthongkum N, Fung HL, Zhang K, Zhang K. Identification of methylation haplotype blocks aids in deconvolution of heterogeneous tissue samples and tumor tissue-of-origin mapping from plasma DNA. Nat Genet 2017; 49:635-642.

9 Shen L, Song CX, He C, Zhang Y. Mechanism and function of oxidative reversal of DNA and RNA methylation. Annu Rev Biochem 2014; 83:585-614.

10 Vasanthakumar A, Godley LA. 5-hydroxymethylcytosine in cancer: significance in diagnosis and therapy. Cancer Genet 2015; 208:167-177.

11 Tahiliani M, Koh KP, Shen Y, et al. Conversion of 5-methylcytosine to 5-hydroxymethylcytosine in mammalian DNA by MLL partner TET1. Science 2009; 324:930-935.

12 Mellen M, Ayata P, Dewell S, Kriaucionis S, Heintz N. $\mathrm{MeCP} 2$ binds to $5 \mathrm{hmC}$ enriched within active genes and accessible chromatin in the nervous system. Cell 2012; 151:1417-1430.

13 Song CX, Szulwach KE, Fu Y, et al. Selective chemical labeling reveals the genome-wide distribution of 5-hydroxymethylcytosine. Nat Biotechnol 2011; 29:68-72.

14 Thomson JP, Lempiainen H, Hackett JA, et al. Non-genotoxic carcinogen exposure induces defined changes in the 5-hydroxymethylome. Genome Biol 2012; 13:R93.

15 Feng J, Shao N, Szulwach KE, et al. Role of Tet1 and 5-hydroxymethylcytosine in cocaine action. Nat Neurosci 2015; 18:536-544.

16 Kriaucionis S, Heintz N. The nuclear DNA base 5-hydroxymethylcytosine is present in Purkinje neurons and the brain. Science 2009; 324:929-930.

17 Globisch D, Munzel M, Muller M, et al. Tissue distribution of 5-hydroxymethylcytosine and search for active demethylation intermediates. PLOS ONE 2010; 5:e15367.

18 Jin S-G, Jiang Y, Qiu R, et al. 5-Hydroxymethylcytosine is strongly depleted in human cancers but its levels do not correlate with IDH1 mutations. Cancer Res 2011; 71:7360-7365.

19 Zhang Y, Liu T, Meyer CA, et al. Model-based analysis of ChIP-Seq (MACS). Genome Biol 2008; 9:R137.

20 Landt SG, Marinov GK, Kundaje A, et al. ChIP-seq guidelines and practices of the ENCODE and modENCODE consortia. Genome Res 2012; 22:1813-1831.

21 Koh W, Pan W, Gawad C, et al. Noninvasive in vivo monitoring of tissue-specific global gene expression in humans. Proc
Natl Acad Sci USA 2014; 111:7361-7366.

22 van der Maaten L, Hinton G. Visualizing data using t-SNE. $J$ Mach Learn Res 2008; 9:2579-2605.

23 Ritchie ME, Phipson B, Wu D, et al. Limma powers differential expression analyses for RNA-sequencing and microarray studies. Nucleic Acids Res 2015; 43:e47.

$24 \mathrm{Su}$ AI, Wiltshire T, Batalov S, et al. A gene atlas of the mouse and human protein-encoding transcriptomes. Proc Natl Acad Sci USA 2004; 101:6062-6067.

25 Huang da W, Sherman BT, Lempicki RA. Systematic and integrative analysis of large gene lists using DAVID bioinformatics resources. Nat Protoc 2009; 4:44-57.

26 Chowdhury B, Cho IH, Hahn N, Irudayaraj J. Quantification of 5-methylcytosine, 5-hydroxymethylcytosine and 5-carboxylcytosine from the blood of cancer patients by an enzyme-based immunoassay. Anal Chim Acta 2014; 852:212217.

27 Sajadian SO, Ehnert S, Vakilian H, et al. Induction of active demethylation and $5 \mathrm{hmC}$ formation by 5 -azacytidine is TET2 dependent and suggests new treatment strategies against hepatocellular carcinoma. Clin Epigenetics 2015; 7:98.

$28 \mathrm{Xu} \mathrm{H}, \mathrm{Zhu} \mathrm{X}, \mathrm{Xu} \mathrm{Z}$, et al. Non-invasive analysis of genomic copy number variation in patients with hepatocellular carcinoma by next generation DNA Sequencing. J Cancer 2015; 6:247-253.

29 Fraley C, Raftery AE. Model-based clustering, discriminant analysis, and density estimation. J Am Stat Assoc 2002; 97:611-631.

30 Liaw A, Wiener M. Classification and regression by randomForest. $R$ News 2002; 2:18-22.

31 Cohen J. A coefficient of agreement for nominal scales. Educ Psychol Meas 1960; 20:37-46.

32 Huang Y, Rao A. Connections between TET proteins and aberrant DNA modification in cancer. Trends Genet 2014; 30:464-474.

33 Langmead B, Salzberg SL. Fast gapped-read alignment with Bowtie 2. Nat Methods 2012; 9:357-359.

34 Li H, Handsaker B, Wysoker A, et al. The sequence alignment/map format and SAMtools. Bioinformatics 2009; 25:2078-2079.

35 Quinlan AR, Hall IM. BEDTools: a flexible suite of utilities for comparing genomic features. Bioinformatics 2010; 26:841-842.

36 Robinson JT, Thorvaldsdottir H, Winckler W, et al. Integrative genomics viewer. Nat Biotechnol 2011; 29:24-26.

37 Thorvaldsdottir H, Robinson JT, Mesirov JP. Integrative Genomics Viewer (IGV): high-performance genomics data visualization and exploration. Brief Bioinform 2013; 14:178-192.

38 Shen L, Shao N, Liu X, Nestler E. ngs.plot: Quick mining and visualization of next-generation sequencing data by integrating genomic databases. BMC Genomics 2014; 15:284.

39 Huang da W, Sherman BT, Lempicki RA. Bioinformatics enrichment tools: paths toward the comprehensive functional analysis of large gene lists. Nucleic Acids Res 2009; 37:1-13.

$40 \mathrm{Wu}$ C, Orozco C, Boyer J, et al. BioGPS: an extensible and customizable portal for querying and organizing gene annotation resources. Genome Biol 2009; 10:R130.

$41 \mathrm{Wu}$ C, Macleod I, Su AI. BioGPS and MyGene.info: organizing online, gene-centric information. Nucleic Acids Res 2013; 
41:D561-D565.

42 Bolger AM, Lohse M, Usadel B. Trimmomatic: a flexible trimmer for Illumina sequence data. Bioinformatics 2014; 30:2114-2120.

43 Kim D, Pertea G, Trapnell C, Pimentel H, Kelley R, Salzberg SL. TopHat2: accurate alignment of transcriptomes in the presence of insertions, deletions and gene fusions. Genome Biol 2013; 14:R36.

44 Trapnell C, Hendrickson DG, Sauvageau M, Goff L, Rinn JL, Pachter L. Differential analysis of gene regulation at transcript resolution with RNA-seq. Nat Biotechnol 2013; 31:4653.

45 Consortium EP. An integrated encyclopedia of DNA elements in the human genome. Nature 2012; 489:57-74.
(Supplementary information is linked to the online version of the paper on the Cell Research website.)

(c) (i)

This work is licensed under a Creative Commons Attribution 4.0 Unported License. The images or other third party material in this article are included in the article's Creative Commons license, unless indicated otherwise in the credit line; if the material is not included under the Creative Commons license, users will need to obtain permission from the license holder to reproduce the material. To view a copy of this license, visit http:// creativecommons.org/licenses/by/4.0/

(C) The Author(s) 2017 